\title{
Geriatrics education and health professions today: responding to critical need
}

\section{O ensino de profissionais em saúde geriátrica hoje: resposta à necessidade crítica}

\section{Flavia Caretta ${ }^{a}$}

"Dipartimento di Geriatria, Neuroscienze e Ortopedia, Facoltà di Medicina e Chirurgia "A. Gemelli",

Università Cattolica del Sacro Cuore, Roma. <flavia.caretta@rm.unicatt.it>.

\section{ARTICLE INFO}

\section{Article history}

Received: 17/09/2015

Accepted: 22/09/2015

\section{Correspondent Author}

Flavia Caretta

Università Cattolica del Sacro Cuore

Roma, Itália

<flavia.caretta@rm.unicatt.it>

(C) 2015 All rights reserved

\section{Editors}

Alfredo Cataldo Neto

Irenio Gomes

\section{INTRODUCTION}

The connection between health and old age is complex and often influenced L by myths and misconceptions. It is argued that old age is equivalent to a time of illness. Medicine itself has endorsed this idea, to such an extent that gerontology has even been defined as the science that traces the downward trends, thus concentrating its attention on the decline of physiological parameters and thereby reinforcing the assumption of "decline and loss" as elements proper to normal aging. ${ }^{1}$

In order to react to this idea, today there is a tendency to excessively accentuate a model of aging in good health or of successful aging, and from this originates a prejudice towards the disabled elderly, which, in the end, is often shared by the aged people themselves.

Actually, health during old age is a complex amalgam of both biology and personal biography. Therefore, one of the characteristics of the aging population is its substantial heterogeneity, which makes it difficult to formulate a schematization.

All this deeply influences not only the approach to the care of elderly patients, but also the investments and research priorities, the nature and content of geriatrics and gerontology, the perceptions and attitudes of public opinion towards aging.

The social consideration of the aged person also reflects on the idea a healthcare professional has of his/her own profession, something that easily leads to a routine approach, deprived of initiative, changes and, above all, incapable of making it possible for elderly to express their own potential.

The geriatrics healthcare professional, in turn, is generally seen within the social imagination as a figure of little importance, as if he/she had chosen a fallback job, which is scarcely considered.

Therefore, it is necessary to intervene even at the level of social attitudes towards the aged people, in order to foster a cultural change, which is able to have an effect also on the training of those who are professionally engaged within the geriatric field.

About it, recently stressed that progress in including geriatric training within curricula across the health professions continues to lag behind need as a 
result of the continuing presence of barriers identified several decades ago. There remains an urgent need for institutional commitment to enhance geriatric education as a component of health professions curricula. $^{2}$

\section{CARE PROCESS}

In order to talk correctly about the care process, it should be considered first the figure of the healthcare professional and secondly the care recipient.

The quality of care depends on technical expertise, but also on the motivation of those who take care of the patient. Motivations on which the personal and social attitudes towards pain, disability and old age definitely affect.

Too often care is reduced to a series of technical tasks; professionals learn to monitor vital parameters and increasingly sophisticated instruments, but they forget about the protagonist, that is to say the person in his/her entirety.

Indeed, medical institutions often limit themselves to treat pathologies as biophysical entities. The biomedical model - which entails the risk of spreading a reductive perspective in medicine - leads to assert that it is not the task of doctors and healthcare professionals to take care of what age and illness mean to the patient, how he/she is experiencing it, which effect it has on his/ her life and emotions and - in the event they exist - on his/her family and social relations.

Again, death may be experienced as a professional failure and the correlative care as a task without qualification: doctors are trained to heal in the sense of the restitutio ad integrum, that is the restoration to original condition.

It is argued then that death is handled by geriatrics because more often than not people die in old age and, above all, from "diseases poor in satisfaction", which are more and more peculiar to the geriatrician, who is "inured to provide care for unpleasant patients".

These ideas, which are pervasive in the social context, have important implications. They might condition, for example, the number of those who would devote themselves to geriatric medicine $*: 3$ in the United States the baby boomers are reaching the age of retirement and will require more and more assistance, however now there is a shortage of geriatric doctors. Students of medicine themselves, at the beginning of their studies ${ }^{4}$, prefer specializations that imply the use of the new technologies applied to medicine, and

\footnotetext{
* The American Geriatrics Society, Report Shows Critical Gap Between Geriatric Training, Demand for Care. Babyboomers counting on long and healthy life may not find doctors wise to their aging bodies.
}

the treatment of acute pathologies in young patients, rather than dedicate themselves to chronic diseases in the elderly, for whom healing is not expected, or face the difficulty of managing comorbidity. Furthermore, it is argued that such a specialization does not offer a professional prestige.

\section{CARE SETTINGS}

Currently, according to studies conducted on the quality of life of patients living in geriatric care facilities, there often emerges a deep psychosocial and existential suffering experienced by many of them. Yet it is common the observation that rarely a diagnosis and a possible help to this suffering are considered in the care practices. ${ }^{5}$

Nevertheless, the psychosocial models of mental health underline that well-being in old age is significantly influenced by many external factors such as social resources, positive and negative life events, and the spiritual dimension. Gerontological literature itself paid attention to the more and more increasing role of religiousness, spirituality, and the personal sense of life situation, as predictive factors for the well-being of the elderly, in particular for the institutionalized elderly person. ${ }^{6,7}$

Losses on the physical and emotional level and the dependence on others are inevitable aspects the patient has to face, but in addition to these, there is often a lack of knowledge and respect for cultural differences and religious convictions, or a lack of meaningful interactions and an inadequate dialogue with healthcare professionals. ${ }^{8}$

Besides the fragility of the elderly, there is the weakness of the institutions, which can be identified in a sense of impotence due to its capacity to foster positive changes, in a lack of uniformity in approaching the patient, a low level of motivation, and a widespread apathy among the staff, which diminishes all efforts and activities. In fact, when an organization absorbs and embraces a pessimistic and fatalistic conception of old age, changes and innovation are no longer sought after.

Geriatrics is defined, with good reason, as the medicine of complexity. It is a complexity that also requires learning to relate with people affected by cognitive deficits and, at least apparently, incapable of giving an answer, as well as facing problems like restraint - which is still considered as a too usual "therapeutic modality" - and abuse, as well as care plans that do not include rehabilitation, occupational therapy, aids, and a reduction of prescription antipsychotic medications. ${ }^{9}$

Many studies denounce the enormously high rate of prescription of antipsychotic medications in nursing 
homes, without any clinical evidence, any reference to guidelines and recommended dosage. It is clear that beneath this way of acting, there is a wrong concept of the aged person and his/her value.

One of the more demanding challenges, due to its healthcare burden, is dementia, an everincreasing disease. Therefore, if a demented person is considered as the "shell" or the "pod" of the former self, this person might be relegated to an undefined zone, beyond a clear concept of person, causing the loss of rights and privileges every human being has. Can it be taken for granted that people working in geriatric field have a different concept? Or that they are able to influence the healthcare world about the unchanged value of the person even in such situations?

Even still, various studies maintain that dementia does not preclude the possibility of finding meaning in one's condition of life if the person in question is supported by interpersonal relations and the proper assistance. $^{10}$

Indeed, personality is the product of relations with others and can be improved or harmed on the basis of how the person is valued or depersonalized, respectively. ${ }^{11}$

\section{DEVELOPMENT OF RELATIONAL SKILLS}

The relational component in healthcare, which is given for granted in healthcare rhetoric, actually tends to be defined as "not work", and therefore is hardly recognized and valued in practice by the organization. The aspect of compassion, loving care and attention to the different needs of the person are considered, with respect to other tasks to be performed, as a "waste of time", "emotional weaknesses", or "non-professional additions".

Nevertheless, the care process cannot be reduced to a protocol made up of procedures. An unpredictable human dimension, which cannot be standardized, is implied in it, and this dimension has to be made the most of, reciprocally, within the personal relation.

The experience of illness and the terminal phases of life cannot be described: they concern the mystery and the most intimate dimension of the person and thus require the healthcare worker a broader concept of care. It is required to acquire the paradigm of complexity, which does not deny the technicalscientific component - with its important evidences on the level of clinical efficacy and organizational efficiency - on the contrary, it is part of, and completes it, by introducing it within the "human" dimension, of which feelings, discretion, uniqueness, and ethical value fully are part. ${ }^{12}$
It becomes necessary, then, a question that organizations, people directing or working within cannot ignore. How should we have to take care of experiences, questions of meaning belonging to those who are at the front line in places of illness, old age and death?

In the current complex healthcare organization, human qualities, motivation and an adequate education (scientific, technical and ethical) of individual health workers are not enough; it is also necessary that the various structures promote organizational actions and training support.

On the other hand, if the care process implies in any case a reciprocity between those providing and receiving care, also the elderly are requested to accustom themselves to new life situations and learn to accept limitations and not to withdraw into themselves. It is obvious that this education to the old age cannot be improvised, rather must start in advance. From this perspective, formation should involve the care recipients themselves.

With respect to healthcare professionals, it is clear that the complexity of geriatric care requires particular requisites, such as the ability to value a relationship with patients that endures through time and to appreciate the challenges stemming from complexity and interdisciplinarity. It also requires them to be aware of their own responsibility, which is social as well, to find professional satisfaction even when faced with apparently limited changes, as well as to prefer the interdisciplinary work. ${ }^{13}$

If a specific training is critical for the good of the relationship with the patient, also a training aimed at realizing interdisciplinary care teams is essential.

Moreover, relation should not be approached merely from the perspective of the patient: what seems to be oriented exclusively towards the care and well-being of the patient is actually of benefit to the healthcare professionals and care teams as well, and often even to the health care institutions.

Each professional experience, both negative and positive, definitely contributes to shaping a medical-scientific competence and furthermore it has consequences on the psychical and relational dimension of the professional.

Hence, in geriatrics, the care objective should be supporting a level of quality of life that respects the dignity, identity and needs of both persons receiving health care and those providing it. ${ }^{14}$

This healthcare model is only possible within a context of trust and in a relation of mutual respect, and thanks to this model, providing care could become an enriching experience. Otherwise, it would become something much similar to "custody". 
A good communication is also therapeutic. Building relationships is the cornerstone of nursing work, particularly with patients with learning disabilities; communication is a prerequisite to that process. ${ }^{15}$

In brief, the relationship with the patient could be defined as communicating on the same wavelength as him, through a continuous exchange of words and expressive nuances, whose tones should aim at adapting, sometimes responding to a mere non-verbal reaction of the patient. This is a message that is science, humanity and art as well. ${ }^{16}$

The communication established with the patient and with his/her relatives - is the most important aspect of inter-professionalism within the health care. For the patient, his/her relatives constitute a fundamental link to the external world, his/her personal biography and values as well. ${ }^{17}$

It is therefore essential to include the patient's family in any professional collaboration, ${ }^{18}$ since an effective communication with the family might even improve the clinical process and the very outcomes. ${ }^{19}$

\section{RELATIONSHIP AND COMMUNICATION WITHIN A CARE TEAM}

The way of relating to the patient is closely linked to the communication with other healthcare professionals: they speak to the patient in the same way they do with their colleagues. Currently there is a tendency, within the care practice, to give more and more importance to the care team, teamwork and multidisciplinarity. After all, it is right the technological progress in the field of medicine that makes working together "obligatory".

Hence, it is required not only the competence of individual professionals, but also the capability to integrate the various competences. In this way, each healthcare professional will be able to acquire a "homogeneous attitude", without any contradictions. With regard to geriatric institutions - which become a place of life, and frequently of death too, for the elderly - a "harmony" among professionals working in them is indispensable. This would make possible for the aged person to choose the interlocutor he/ she prefers, someone who is more suitable for his/her sensitivity.

Are the health professionals prepared for this? The training process is still oriented towards an "autonomous" way of working: consequently in the various care places is observed individualism, esprit de corps, defense of one's own role, and the tendency to aim at a successful career also to the detriment of colleagues. These are aspects that predispose professionals to burnout, which is defined not only as something determined by individual factors but also as a dysfunction of the organization.

Yet a team cannot be improvised, and interdisciplinarity does not simply take shape because professionals work together in the same place and for the same objectives. One of the conditions is that all members must accept that no one knows the answer to any question, given that everyone somehow depends on the culture and competence of others. The result, then, is not a simple sum of individual professional competences, rather an original and shared response, a synergy of perspectives and proposals that shall be able to really consider the patient as a person in all of his/her dimensions.

It is right in such openness to the contribution of colleagues that the change from multidisciplinarity to interdisciplinarity may happen. Yet to make this possible it is necessary to approach others with an attitude to learn, in order to discover what kind of contribution and enrichment colleagues may offer. Sometimes, at the workplace, people instinctively look at their colleagues and other professionals while supposing everyone's limits, or thinking about any difficulties experienced in their relationship with them. It is precisely this approach that impedes any potential, changes, or attitude of understanding to emerge.

Therefore, it is evident that a team cannot be conceived simply from the perspective of the patient, rather, it should be considered first from the perspective of the healthcare professionals. Team is the first "place" where professionals may elaborate psychological and spiritual burdens that a healthcare work could imply with the passing of time. The possibility to share with others one's own emotional involvement when faced with a particularly painful situation, to dialogue about difficult decisions, or ask for an opinion, is a fundamental help and reveals itself to even be "therapeutic" for the healthcare professional. A review of the literature published between 1985 and $2004^{20}$ compares healthcare environments where people work in a team and the common ones (without teamwork). In conclusion, the elements that most influence professionals' satisfaction and the perception of the team effectiveness were collaboration, participation, cohesion, and the resolution of conflict.

Conflict - as in all interpersonal relations might be inevitable even in professional relations. In professional relations, in order to explain dysfunction and conflict there is the tendency to attribute them to possible incompatibility of character, to any problematic aspects of personality, negative aspects of the work 
environment, or to the stress originated from the work performed. These all are factors that might influence conflict, but the way of communicating and relating to the patient, colleagues and the institution is rarely considered as the cause of such difficulties.

Working together means operating according to certain choices and decisions, which are influenced by people's own perception, values, interests and expectations $^{21}$. If situations of conflict are at times unavoidable, one could at least try to face them as opportunities for personal growth and finding new ways of inter-relation. This happens, for example, by trying to agree on common objectives, learning to overcome personal impressions, in order to acknowledge those of others. Furthermore, it was found that the teamwork could also lead to an improvement of the outcomes of functional health, as well as to a reduction of the duration of hospitalization, and a lower turnover of personnel ${ }^{22}$.

\section{CONCLUSION}

To achieve a future health care workforce attuned to addressing the needs of an older population, it is imperative that we move from grudging, glacier like acceptance of the need for geriatric and gerontological education toward enthusiastically embracing such education as a societal priority that must be met regardless of cost and profitability. ${ }^{2}$

In addition to the ethical duty to get an ongoing scientific education, healthcare workers need to receive a human formation and preparation, especially in a cultural moment where old age, suffering and death seem to have lost their meaning.

In all areas of medicine and healthcare, it is important to plan interdisciplinary training initiatives, and this is even more important in the geriatric field. Indeed, precisely because of the intrinsic characteristics of geriatrics and the necessity of a global approach to the person, it was stated that geriatric medicine is well suited to become "the soul of medicine". ${ }^{23}$

What is the commitment required to healthcare organizations and the individual professionals?

An ongoing formation should start by checking the professional competences and the educational curriculum of each worker (even the one to enter into profession), in order to develop communication skills and the "technical" updating as well. Organizations, too, are to be regenerated as a physical and symbolic space that supports interaction and generates attention towards persons, asserts shared values, gives a possibility to think, discuss, make decisions and take initiatives for change. ${ }^{12}$
This capacity to transform is an indication of the effectiveness of any educational path and strongly motivates professionals to work well even in care relationships, which always involve educational processes, both in the healthcare worker-patient relation and within the team.

The very exercise of profession should be understood as a search for solutions arising from one's own ideas and professional competence, yet always in dialogue with others for the benefit of one's own work as well as of the overall organization. A person, independently from his/her role or function within the society, should in any case feel responsible for change. All this allows, then, to realize a care environment that is really "therapeutic" for anyone: patients feel a climate of serenity, attention, listening and care, in its fullest sense; workers feel appreciated and valued for their work; and those holding leadership roles will experience less the weight of responsibilities and decisions, since they will be taken and shared by the entire personnel. A further result will be a continuous increase in the quality standards, not only in complying with quality controls of evaluation protocols, but also in making this quality as a real "well-being" for all those involved in the care process.

\section{REFERENCES}

1. Caretta F. Una società per tutte le età. La persona anziana risorsa per un mondo unito. in Atti Congresso Internazionale Movimento Umanità Nuova. Roma: Città Nuova Editrice; 1997.

2. Bardach SH, Rowles GD. Geriatric Education in the Health Professions: Are We Making Progress? Gerontologist. 2012;52(5):607-18.

3. Diachun LL, Hillier ML, Stolee P. Interest in Geriatric Medicine in Canada: How Can We Secure a Next Generation of Geriatricians. J Am Geriatr Soc. 2006;54(3):512-9.

4. Shue CK, McNeley K, Arnold L. Changing medical student's attitudes about older adults and future older patients. Acad Med. 2005;89(10):S6-9.

5. Forbes-Thopson S. Nursing Homes and Suffering: Part of the Problem or Part of the Solution. J Appl Gerontol. 2006;25(3):234-51.

6. Fry PS. Religious involvement, spirituality and personal meaning for life: existential predictors of psychological wellbeing in community-residing and institutional care elders. Aging Ment Health. 2000;4(4):375-87.

7. Crowther MR, Parker MW, Achenbaum WA, et al. Rowe and Kahn's Model of Successful Aging Revisited. Gerontologist. 2002;42(5):613-20.

8. Dwyer LL, Nordenfelt L, Ternestedt BM. Three Nursing Home Residents Speak About Meaning At the End of Life. Nurs Ethics. 2008;15(1):97-109.

9. Briesacher AB. The Quality of Antipsychotic Drug Prescribing in Nursing Home. Arch Inter Med. 2005;165(11):1280-5.

10. Jennings B. Alzheimer's disease and the quality of life. In: Doka KJ, editor. Living with Grief: Alzheimer's Disease. New York: Springer; 2004. p. 257-8. 
11. Chenoweth L, King MT, Jeon YH, et al. Caring for aged dementia care resident study (CADRES) of person-centred care, dementia-care mapping, and usual care in dementia: Acluster randomized trial. Lancet Neurol. 2009;8(4):317-25.

12. Orlando I. Prevenzione del burnout e lavoro di équipe. In: Società Italiana di Gerontologia e Geriatria. Le cure di fine vita in geriatria. Firenze: Società Italiana di Gerontologia e Geriatria; 2008.

13. Howe JL, Scherman DW. Interdisciplinary educational approaches to promote team-based geriatrics and palliative care. Gerontol Geriatr Educ. 2006;26(3):1-16.

14. Bernabei R, Caretta F, Petrini M. Aspetti gestionali e bioetica, interventi non farmacologici sul paziente e azioni di sostegno del caregiver. In: Le demenze in Italia. Casi clinici interattivi. Torino: UTET; 2006.

15. Collins S. Good communication helps to build a therapeutic relationship. Nurs Times. 2009;105:24.

16. Fernandez C. The Ethics of Communication in Medicine. Ital Hearth J. 2002;3(12):1225-8.
17. Lopez RP. Suffering and Dying Nursing Home Residents: Nurses' Perceptions of the Role of Family Members. J Hosp Palliat Nurs. 2007;9(3):141-9.

18. Thompson DE. Interprofessionalism in Health Care: Communication with the Patient's Identified Family. J Interprof Care. 2007;21(5):561-3.

19. Bluestein D, Latham Bach P. Working with Families in Long Term Care. J Am Med Dir Assoc. 2007;8(4):265-70.

20. Lemieux C, Mc Guire WL. What Do We Know About Health Care Team Effectiveness? A review of the literature. Med Care Res Rev. 2006;63(3):263-300.

21. Youngwerth J, Twaddle M, Cultures of interdisciplinary teams: how to foster good dynamics. J Palliat Med. 2011;14(5):650-4.

22. Gelb Safran D, Miller W, Beckman H. Organizational dimensions of relationship-centered care. J Gen Intern Med. 2006;21:S9-15.

23. Elon RD. Perspectives on the future of geriatric medicine. J Am Med Dir Assoc. 2006;7(3):197-200. 